\title{
Why proton temperature and velocity are correlated in the SW and not in ICMEs?
}

\author{
Pascal Démoulin \\ Observatoire de Paris, LESIA, UMR 8109 (CNRS), F-92195 Meudon Principal Cedex, France
}

\begin{abstract}
The in situ correlation of the proton temperature with the bulk plasma velocity of the solar wind (SW) is a firm result confirmed by several spacecraft, while in interplanetary coronal mass ejections (ICMEs) the proton temperature is both cooler and not correlated with the velocity. For some specific heating mechanism, the internal energy equation alone could provide a temperature increasing with velocity, but the increase remains much weaker than observed in the SW. In fact, it is shown here that the observed correlation is generically obtained from a global energy conservation in thermally driven winds. It results from the conversion of thermal to kinetic energy close to the Sun. The absence (or even anti-) correlation observed in situ between electron temperature and SW velocity, together with the correlation found for proton temperature, show that protons have a dominant role in the SW acceleration. In contrast, in ICMEs the plasma is contained by the magnetic field since ICMEs have both a closed configuration and a low plasma $\beta$. It implies no significant correlation between temperature and velocity, as observed. In conclusion, a different dominating term in the impulsion equation is the main origin of the different relation observed between the proton temperature and the bulk velocity in SW and ICMEs.
\end{abstract}

Keywords: Coronal Mass Ejections, Magnetic cloud, Magnetic field, Solar Wind

PACS: $96.50 . \mathrm{Ci}, 96.50 . \mathrm{Uv}, 96.60 . \mathrm{Vg}$

\section{INTRODUCTION}

Just after the discovery of the SW by spacecraft, a correlation was discovered between the outward velocity, $v$, and the proton temperature, $T_{p}[1,2]$. Several functions $T_{p}(v)$ have been fitted to the $\mathrm{SW}$ data $[$ e.g. 3, 4]. More recently, Elliott et al. [5] removed the ICMEs from the SW data, and found a different linear law for the compression and rarefaction regions. They also found no significant variation of the proton temperature with the ICME velocity (figure 1 ).

The observed $T_{p}(v)$ correlation was found in an exospheric SW model by [6] (at least for the slow SW, see their figure 12). Rather, the $T_{p}(v)$ correlation was interpreted with the variation of the estimated polytropic index within different range of the measured SW velocity [3, 7]. Recently, Matthaeus et al. [8] proposed a theoretical mechanism to explain this correlation.

The observed $T_{p}(v)$ correlation is such a strong result that it is recurrently used to define the expected $T_{p}$ from the measured bulk velocity. One criteria to identify ICMEs is a measured $T_{p}$ lower than half the expected $T_{p}$ [9]. ICMEs are formed by the ejection from the Sun of magnetized plasma during CMEs [e.g. 10, 11].

The main objective of the present work is to understand the physical origin of the $T_{p}(v)$ correlation in the SW with a simple one fluid model, following [12]. A quantitative comparison of the model proposed by [8] to the data shows that $T_{p}$ is increasing too slowly with $v$ (section 2). In fact, the acceleration phase of the SW should be taken into account. This does provide gener- ically a $T_{p}(v)$ dependence comparable to observations, then the model is extended to a bi-fluid model (section 3 ). Then, the same approach explains the absence of significant dependence of $T_{p}$ on $v$ in ICMEs (section 4).

\section{2. $T_{p}(v)$ : DUE TO SPECIFIC HEATING?}

Let us consider a stationary flow with all variables depending only on the distance, $r$, to the Sun. With $T_{p}=$ $T_{e}=T$, the internal energy equation is

$$
\frac{3}{2} \frac{\mathrm{d} T}{\mathrm{~d} r}-T \frac{\mathrm{d} \ln \rho}{\mathrm{d} r}=\frac{m_{\mathrm{p}}}{2 k_{\mathrm{B}}}\left(\frac{Q}{v}-\frac{\mathrm{d} q /(\rho v)}{\mathrm{d} r}\right)
$$

where $Q$ is the heating per unit time and mass, $q$ is the heat flux per unit surface and time.

Matthaeus et al. [8] proposed that the observed $T_{p}(v)$ relationship is due to a specific property of $Q$ in the region far from the Sun where $\rho \propto r^{-2}$ and where $v$ is almost independent of $r, v=U$. Then, the variable $\zeta=r / U$ is approximately measuring the "age" of the plasma since its departure from the Sun. With the above hypothesis, as well as $Q(\zeta)$ and $q=0$, equation (1) is rewritten as

$$
\frac{\mathrm{d} T}{\mathrm{~d} \zeta}+\frac{4}{3} \frac{T}{\zeta}=\frac{m_{\mathrm{p}}}{3 k_{\mathrm{B}}} Q(\zeta)
$$

which has the following general solution [12]

$$
T(\zeta)=\frac{m_{\mathrm{p}}}{3 k_{\mathrm{B}}} \zeta^{-4 / 3} \int_{\zeta_{o}}^{\zeta} Q \zeta^{4 / 3} \mathrm{~d} \zeta^{\prime}+T_{o}\left(\frac{\zeta}{\zeta_{o}}\right)^{-\frac{4}{3}}
$$




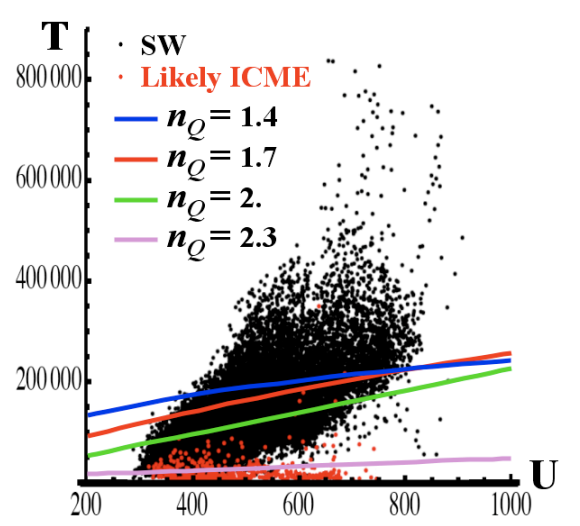

FIGURE 1. Comparison of the results given by equation (4) with the results of [8]. The data points are reproduced from their paper. $T$ is the proton temperature and $U$ is the velocity; both are measured by the ACE spacecraft. ICMEs and their surroundings are removed in the SW data points (black).

where $T_{o}$ is the temperature for $\zeta_{o}=r_{o} / U$, and $r_{o}$ is a reference position close to the Sun.

Since the wind has a uniform velocity $(U)$, we have $\zeta / \zeta_{o}=r / r_{o}$, so the last term in equation (3) is function only of $r / r_{o}$. In contrast to [8], the conclusion is that there is no correlation between $T$ and $U$ for an adiabatic evolution, unless there was already a correlation close to the Sun [so $\left.T_{o}(U)\right]$.

A correlation $T(U)$ is created by the first term in equation (3) since it depends on $\zeta$ (and not only on $\zeta / \zeta_{o}$ ). For a given distance $r$, this implies in general $T(U)$. Let us express this correlation with $Q(\zeta)=Q_{1} \zeta^{-n_{\mathrm{Q}}}$, where $Q_{1}$ and $n_{\mathrm{Q}}$ are constants, then

$$
T(\zeta)=T_{1}\left[\zeta^{1-n_{\mathrm{Q}}}-\left(\frac{\zeta}{\zeta_{o}}\right)^{-\frac{4}{3}} \zeta_{o}^{1-n_{\mathrm{Q}}}\right]+T_{o}\left(\frac{\zeta}{\zeta_{o}}\right)^{-\frac{4}{3}}
$$

with $T_{1}=m_{\mathrm{p}} Q_{1} /\left(k_{\mathrm{B}}\left(7-3 n_{\mathrm{Q}}\right)\right)$.

Between 0.3 and $5 \mathrm{AU}$, the mean electron and proton temperature varies typically between $r^{-1}$ and $r^{-0.7}$, then, the adiabatic term in equation (4) is unable to explain such gradients. Rather, for $r / r_{o}>>1$, the temperature profile is dominated by the first term, so that $n_{\mathrm{Q}}$ is in the typical range $1.7 \leq n_{\mathrm{Q}} \leq 2$. Moreover, the approximate power law observed for $T(r)$ justify the simple form of $Q$ given above as a first-order estimation.

With $n_{\mathrm{Q}}$ within the range $1.7 \leq n_{\mathrm{Q}} \leq 2$, the temperature predicted by equation (4) has a much lower slope than for the SW data (figure 1). Extending the range of $n_{\mathrm{Q}}$ to lower values simply provides an even lower slope. Increasing $n_{\mathrm{Q}}$ does provide a large dependence of $T$ on $U$ for the first term in equation (4), but as $n_{\mathrm{Q}}$ gets closer to $7 / 3$, this effect is masked by a larger contribution of the adiabatic term.

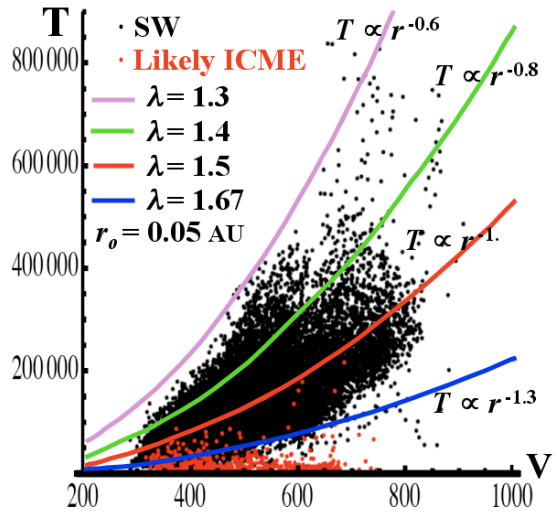

FIGURE 2. Comparison of the results given by equation (9) with the results of [8]. $\lambda$ is the polytropic index, and $r_{o}$ is the distance from the Sun where the polytropic law is supposed to begin. The radial dependance of the temperature for $r>>r_{o}$ is indicated.

In conclusion, assuming $Q(r / U)$ does provide a positive correlation between $T$ and $U$, but the implied slope in this correlation is significantly lower than observed. The observed correlation would require a heating term of the form $U^{e} \zeta^{-n_{\mathrm{Q}}}$ with the exponent $e$ typically in the interval $[2,3]$. So this would require an ad hoc and an important deviation from the concept proposed by [8].

Inside the inner heliosphere $(r<1 \mathrm{AU})$, the proton temperature is typically decreasing as $r^{-n_{\mathrm{T}}}[13,7]$. This observational result can be incorporated in a model with a polytropic law:

$$
T_{p}=T_{p, o}\left(\rho / \rho_{o}\right)^{\lambda_{p}-1},
$$

where $\lambda_{p}$ is the polytropic index. This provides a simple solution of the internal-energy equation. Equation (5) provides a $T_{p}(v)$ correlation if $\lambda_{p}$ is function of $v$. However, such correlation was not found in the inner heliosphere. The data rather show that the $T_{p}(v)$ correlation observed at $1 \mathrm{AU}$ has almost the same form at least as close as $0.3 \mathrm{AU}$ [see figure 3 in 13]. This indicates that the origin of the $T_{p}(v)$ correlation is closer to the Sun.

\section{CORRELATION $T_{p}(v)$ IN THE SW}

For a radially-symmetric and stationary SW flow the radial projection of the momentum equation is

$$
v \frac{\mathrm{d} v}{\mathrm{~d} r}=-\frac{\mathrm{d} P / \mathrm{d} r}{\rho}-\frac{G M_{\odot}}{r^{2}}+f_{r},
$$

where $f_{r}$ incorporates any extra force per unit mass. In contrast to the analysis done in section 2 , the velocity $v$ is explicitly a function of $r$, while $T_{p}=T_{e}=T$. 

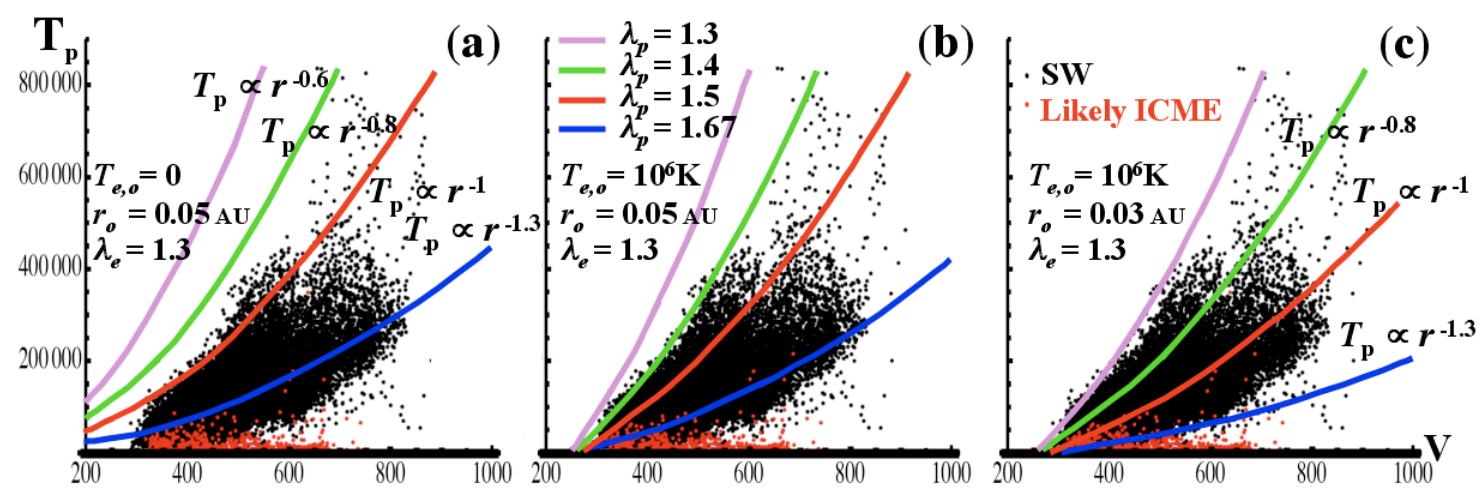

FIGURE 3. Comparison of the results given by equation (11) with the results of [8]. The data points are reproduced from their paper. $\lambda$ is the polytropic index, and $r_{o}$ is the distance from the Sun, in AU, where the polytropic law is supposed to begin. The radial dependance of the temperature for $r>>r_{o}$ is indicated.

The integration of equation (6) from $r_{o}$ to $r$, provides a generalized Bernoulli equation

$$
\frac{v^{2}}{2}+\int_{r_{o}}^{r} \frac{\mathrm{d} P / \mathrm{d} r}{\rho} \mathrm{d} r-\frac{G M_{\odot}}{r}-\int_{r_{o}}^{r} f_{r} \mathrm{~d} r=\frac{v_{o}^{2}}{2}-\frac{G M_{\odot}}{r_{o}} .
$$

The temperature is mostly given by equation (1) with only a weak effect of the velocity (section 2 ). The velocity is mostly given by the Bernoulli equation. The term $\int_{r_{o}}^{r} \frac{\mathrm{d} P / \mathrm{d} r}{\rho} \mathrm{d} r$ is approximately proportional to the temperature (with a distance weighting within the integral). This is the main origin of the $T_{p}(v)$ correlation: with a higher temperature, the wind is accelerated to higher velocity. This is a general property of thermally driven winds.

The above property is illustrated with a polytropic law. With equation (5), equation (7) is rewritten as

$$
\frac{v^{2}}{2}=\frac{k_{\mathrm{B}}}{m_{\mathrm{p}}} \frac{2 \lambda}{\lambda-1}\left(T_{o}-T\right)+C,
$$

where $C$ collects all the terms that do not depend on $v$ or $T$ (so we suppose $f_{r}$ independent of $v$ and $T$ to stick to the basic argumentation). With $\rho \propto r^{-2}$, and for $r>r_{o}$, equation (8) is rewritten as

$$
T(r, v)=\frac{\lambda-1}{2 \lambda}\left(\frac{r}{r_{o}}\right)^{-2(\lambda-1)} \frac{m_{\mathrm{p}}}{k_{\mathrm{B}}}\left(\frac{v^{2}}{2}-C\right) .
$$

For a fixed distance $r$, and a given $\lambda, T$ is a quadratic function of $v$. The comparison to the data is shown in figure 2 for the simple case $C=0$, a case where the acceleration provided by $f_{r}$ is just compensating for the difference of gravitation potential. Cases with $C \neq 0$ are simply deduced by translation of the curves along the $v$-axis when $C$ is independent of $T$ and $v$. If some acceleration model provides such dependence then the curves are deformed accordingly.

It is remarkable that such a simple approach provides the main trend present in the observations without inputting extra hypothese. Fixing the parameters, $\lambda, r_{o}$, and
$C, T(v)$ has a quadratic dependence as observed if we consider the global distribution of the data points (figure 2). Moreover, the above polytropic model also permits to interpret the increase of $T$ dispersion with an increasing $v$, with variable polytropic index $\lambda$.

However, most of the SW data points are grouped in a narrower region. Elliott et al. [5] concluded that a linear fit represents well the mean tendency of $T(v)$ for the full range of $v$. Their linear fit are around the curve $\lambda=1.5$ in figure 2 . It is remarkable that this correspond to a typical value of the temperature gradient in the SW, $n_{\mathrm{T}} \approx 1$, independently deduced from other observations. Moreover with the observed dispersion in $T$, one can hardly distinguish the curves around $\lambda=1.5$ from a linear dependance. Then, equation (9) is also compatible with the mean linear tendency found by [5].

The above derivation can be generalized to a multi fluid model. Let us consider only electrons and protons. Due to their large difference of mass and low level of collisions in the SW electrons and protons are expected to have a low exchange rate of energy, so different temperatures, $T_{e}$ and $T_{p}$, as observed [e.g. 14]. Equation (7) is simply modified with $P$ replaced by $P_{e}+P_{p}$. Assuming a polytropic law for both species, so equation (5) with $\lambda_{e}$ and $\lambda_{p}$, as supported by observations [13], equation (8) is transformed to

$$
\frac{v^{2}}{2}=\frac{k_{\mathrm{B}}}{m_{\mathrm{p}}}\left(\frac{\lambda_{e}\left(T_{e, o}-T_{e}\right)}{\lambda_{e}-1}+\frac{\lambda_{p}\left(T_{p, o}-T_{p}\right)}{\lambda_{p}-1}\right)+C .
$$

The dominant contributions are given by the temperatures $T_{e, o}$ and $T_{p, o}$. If the electrons would be dominant in accelerating the SW, then equation (10) would imply a quadratic $T_{e}(v)$ relationship, while no or even a negative correlation is observed [e.g. 15]. Indeed coronal observations indicate that $T_{p, o}$ is greater than $T_{e, o}$ by a factor 2 to 3 [e.g. 16]. Then, equation (10) implies no significant link between $T_{e}$ and $v$. 
As above, with $\rho \propto r^{-2}$, and for $r>>r_{o}$, equation (10) is rewritten as

$$
\begin{aligned}
T_{p}(r, v)= & \frac{\lambda_{p}-1}{\lambda_{p}}\left(\frac{r}{r_{o}}\right)^{-2\left(\lambda_{p}-1\right)} \\
& \times\left(\frac{m_{\mathrm{p}}}{k_{\mathrm{B}}}\left(\frac{v^{2}}{2}-C\right)-\frac{\lambda_{e} T_{e, o}}{\lambda_{e}-1}\right) .
\end{aligned}
$$

With $T_{e, o}<<T_{p, o}$, equation (11) implies a quadratic $T_{p}(v)$ relationship, but with $T_{p}$ twice larger than with a single fluid (compare figures 2 and $3 a$ ). With a typical coronal temperature $T_{e, o}=10^{6} \mathrm{~K}$, the model is closer to the data for low velocities (figure $3 b$ ). Finally, the main trend of the data is reproduced by this simple bi-fluid model with a polytropic law starting closer to the Sun (figure 3c).

\section{NO $T(v)$ CORRELATION IN ICMES}

The properties of plasma measured in ICMEs are significantly different than in the SW [e.g. 17]. Moreover, not only is the proton temperature much lower in ICMEs than in the SW, but also the correlation $T(v)$ disappears as reproduced in figures 1-3 from the results of $[5,8]$.

Is the heating mechanism different or in a different regime? This is, a priori, plausible, in view of the very different characteristics measured in the SW and ICMEs, as follow. In the SW, the magnetic field is variable and the plasma- $\beta$ fluctuates around unity. In contrast, a wellorganized magnetic field with low fluctuations, and a low $\beta(\leq 0.1)$ are observed in ICMEs. Indeed, figure 1 shows that heating with $n_{Q} \approx 2.3$ could explain $T_{p}(v)$ observed in ICMEs. However, this hypothesis, of an heating decreasing with distance faster than in the $\mathrm{SW}$, is not supported by the similar power laws found for $T_{p}(r)$ and $T_{e}(r)$ in the SW and ICMEs [e.g. 17].

Rather, in the line of thought of section 3, the momentum equation is also one of the main equations governing the physics of ICMEs. However, the dominant terms, as well as the geometry of the magnetic field, are different in the SW and in ICMEs, and it is worth analyzing the consequences.

The main difference is that SW magnetic field lines are open, while ICME field lines are dominantly closed and still connected to the Sun $[$ e.g. 18, 19]. Let us consider different winds with different heating. As the heating increases, the temperature and the plasma pressure increases, accelerating the plasma to higher velocities along the open magnetic field lines. The same happens with a larger deposition of momentum. However, in a closed magnetic configuration, this cannot happen, unless the plasma pressure becomes high enough so that the magnetic configuration is blown up towards an open magnetic-field configuration. In ICMEs, this last case could not happen, since the plasma $\beta$ is significantly less than unity.

Let us summarize further the physics of ICMEs, in order to contrast it with the one present in the SW. The general momentum equation applies to both, but equation (6) applies only to the SW, since the main term in ICMEs, the Lorentz force, is neglected in equation (6). Indeed, this magnetic force, together with the contribution of a drag force, determine the temporal evolution of the velocity in ICMEs, in good agreement with the observed velocity of CMEs/ICMEs in the inner heliosphere [e.g. 20]. Then, the evolution of the field configuration and of the main velocity components (expansion and velocity of the mass centre) of ICMEs, are expected to be nearly independent of the thermodynamics of the ICME plasma. It implies that no significant correlation between $T$ and $v$ is expected in ICMEs, as observed.

\section{REFERENCES}

1. L. F. Burlaga, and K. W. Ogilvie, Astrophys. J. 159, 659-670 (1970).

2. A. J. Hundhausen, S. J. Bame, J. R. Asbridge, and S. J. Sydoriak, J. Geophys. Res. 75, 4643-4657 (1970).

3. R. E. Lopez, and J. W. Freeman, J. Geophys. Res. 91, 1701-1705 (1986).

4. M. Neugebauer, J. T. Steinberg, and R. L. Tokar, et al., Space Sci. Rev. 105, 661-679 (2003).

5. H. A. Elliott, D. J. McComas, and N. A. Schwadron, et al., J. Geophys. Res. 110, A04103 (2005).

6. J. Lemaire, and M. Scherer, Rev. Geo. Space Phys. 11, 427-468 (1973).

7. T. L. Totten, J. W. Freeman, and S. Arya, J. Geophys. Res. 100, 13-17 (1995).

8. W. H. Matthaeus, H. A. Elliott, and D. J. McComas, J. Geophys. Res. 111, A10103 (2006).

9. J. G. Richardson, and H. V. Cane, J. Geophys. Res. 100, 23397-23412 (1995).

10. R. F. Wimmer-Schweingruber, N. U. Crooker, and A. Balogh, et al., Space Sci. Rev. 123, 177-216 (2006).

11. T. H. Zurbuchen, and I. G. Richardson, Space Sci. Rev. 123, 31-43 (2006).

12. P. Démoulin, Solar Phys. 257, 169-184 (2009).

13. E. Marsch, K. M. Thieme, H. Rosenbauer, and W. G. Pilipp, J. Geophys. Res. 94, 6893-6898 (1989).

14. N. Meyer-Vernet, Basics of solar wind, Cambridge University Press, Cambridge, 2007.

15. G. Gloeckler, T. H. Zurbuchen, and J. Geiss, J. Geophys. Res. 108, A01158 (2003).

16. E. Landi, and S. R. Cranmer, Astrophys. J. 691, 794-805 (2009).

17. Y. Liu, J. D. Richardson, and J. W. Belcher, Planetary Spa. Sci. 53, 3-17 (2005).

18. N. U. Crooker, and T. S. Horbury, Space Sci. Rev. 123, 93-109 (2006).

19. G. D. R. Attrill, L. van Driel-Gesztelyi, and P. Démoulin, et al., Solar Phys. 252, 349-372 (2008).

20. B. Vršnak, Annales Geophysicae 26, 3089-3101 (2008). 